\title{
Conceptualization of an AI Based Assistant to Support the Compatibility of Family and Paid Work
}

\author{
Philippe Krajsic \\ Institute for Applied Informatics (InfAI) \\ Goerdelerring 9, 04109 Leipzig, Germany \\ Email: krajsic@infai.org \\ Bogdan Franczyk \\ Uniwersytet Ekonomiczny we Wroclawiuul \\ Komandorska 118/120, 53-345 Wroclaw \\ Email: bogdan.franczyk@ue.wroc.pl
}

\author{
Martin Schieck, Ingolf Römer, Silvia Torres Landaverde \\ Information Systems Institute \\ Leipzig University \\ Grimmaische Straße 12, 04109 Leipzig, Germany \\ Email: \{schieck, roemer, torres\}@wifa.uni-leipzig.de
}

\author{
Carsta Militzer-Horstmann \\ WIG2 Institut \\ Scientific Institute for Health Economics \\ and Health System Research \\ Markt 8, 04109 Leipzig, Germany \\ Email: carsta.militzer-horstmann@wig2.de
}

\begin{abstract}
The goal of the presented intelligent AI-based assistant is to simplify the reconciliation of paid work and family. In times of the corona pandemic and the challenges it poses, solutions are needed to mitigate the impact on workers and families and to strengthen the compatibility of work and family life. We are developing a conceptual reference model of proven methods and technologies for the generation of information that can be queried by interested parties via an bot service, thus simplifying professional and family challenges. Essentially, we provide a framework and starting point for research, development, and evaluation of an AI-based chatbot for the reconciliation of paid work and family.
\end{abstract}

Index Terms-Artificial Intelligence, Chatbot, BERT.

\section{INTRODUCTION}

$\mathbf{I}$ $\mathrm{N}$ HARDLY any other sector the reconciliation of family and professional obligations is as difficult as in the care and medical services. The demands on personnel in the modern health care system are higher than ever before. In addition to alternating shift work and immense psychological and physical strain at work, most of them also bear responsibility in the private sector-whether it is child care or caring for relatives, possibly made even more difficult by their own illness, pregnancy or separation from their partner. Particularly in respect of the current pandemic situation (Corona Virus / COVID19) and the associated strain on personnel in the care and health sector, this topic is more relevant than ever before. As this is most likely to lead to a permanent change in the way the care and health sector works, and as the reconciliation of paid work and family life brings with it lasting challenges, particularly in this area, solutions must be designed to ease the burden on those involved in this sector. The work presents a technical approach that should be communicated to the scientific community as soon as possible in order to solve

This work was funded by the European Social Fund as well as co-financed by tax revenues on the basis of the budget adopted by the Saxon State Parliament within the project PFaDi — Platform for Family-Related Services the described challenges. Starting from this initial situation of a difficult compatibility of the different areas of life of the described group, this research project is looking for a databased answer, which provides individual, fast, and qualified support in every life situation. This investigation is based on the research project PFaDi (Platform for Family-Related Services). Thanks to bookable services for all situations in life, a unifying calendar function as well as information and links to official applications, guides, and contact addresses, this online platform makes it possible to coordinate the everyday life of families and to support the compatibility of family and professional obligations.

The progress made in the field of intelligent technologies has generated high social expectations. For example, intelligent text and speech recognition have gained high acceptance due to their low threshold (e.g., Alexa). Chatbot applications slick interactions between services and people, improving customer experience. Simultaneously, chatbots offer companies various opportunities to enhance the customer's loyalty and ensure operational efficiency by minimizing the surplus cost of customer service. Constant availability, quick answers and ease of use also contribute significantly to their attractiveness [1], [2].

For a dynamic information generation, the following research question emerges: How could an approach for an appropriate architecture for dynamic interactions in a chatbot context look like?

The remainder of the paper is structured as follows: Section II presents the initial situation in the area of reconciling family and paid work. Section III presents the related work in the area of artificial intelligence, text classification, and regulatory hurdles. In Section IV the reference model and its high-level components are described. In section $\mathrm{V}$ the main component of the reference model, BERT, is described in detail. Section VI explains the pre-training of the BERT model. The final section VII concludes the paper and presents future work. 


\section{INITIAL SITUATION}

Family and paid work can then be regarded as compatible if the conditions allow the acting person to delimit a stableflexible system of areas of action, in which there is scope for planning the transitions between areas of action; compatibility is thus essentially influenced by the ability to regulate the areas or the scope for action, time, and decision-making given there [3]. The issue of reconciling paid work and family/care is often dealt with in the context of the so-called work-life balance. This concept, which deals with the balance between paid work and the rest of life, explicitly includes the area of leisure time and is therefore somewhat broader in scope [4]. Reconciling paid work and family life can also be seen in the context of achieving greater gender equality in the company. While more men are involved in child care and upbringing today than in previous decades, a large part of family and care work is still performed by women. Women are thus exposed to a greater double burden than men in pursuing their professional careers. Family and care work not only contributes to the fact that women are less likely to work, it also makes it more difficult to realise career opportunities. The latter is expressed above all in the fact that women are underrepresented in management positions [5]. For this study, it must be noted that according to a WHO analysis of March 2019 on the gender distribution in the health sector in 104 countries, $70 \%$ of workers are women [6]. Thus, the vast majority of workers in the health sector are women, who experience a particular double burden of combining domestic responsibilities and the specific challenges of their working environment.

In the context of the health care system, the issue of reconciling family and paid work life is important because questions of the quality of health care are affected at different levels. This concerns, among other things, the specific forms of requirements and burdens of the professions working in the health care system, such as shift work, the individual satisfaction and performance of doctors, professional nurses, assistant professions, or therapeutic professions (e.g., physiotherapy, occupational therapy, psychotherapy) or also the guarantee of sufficient (medical) care density in rural areas depending on the availability of a family-friendly infrastructure. Studies from Australia, France, Malaysia, the Netherlands and Hungary show that among physicians of different specialties, the experience of poor work-life balance is positively associated with burnout, but also correlates with other unfavorable outcomes such as willingness to quit or impairment of subjective health [7]. International studies have shown that for professional nurses, conflicts over the balance of paid work and family life are associated with a stronger negative mood, emotional exhaustion, the intention to quit or change jobs, but also with worsening health parameters (pain symptoms, increased cardiometabolic risk score) [7]. In a survey of 120 physicians, a group of researchers around Jerg-Bretzke (2018) revealed that $97.4 \%$ would like to be able to take a break from paid work in case of a family emergency, part-time arrangements, $(96.6 \%)$ and emergency childcare $(90.4 \%)$. In addition, the doctors surveyed demanded that their executives provide more support of compatibility issues $(94.0 \%)$ [8].

In questions of the compatibility of family, care, and paid work, it is possible to draw on already well-developed social research. In addition to general brochures and official forms, there are numerous rigid information portals (e.g., https://www.bmfsfj.de) and websites (e.g., https://care.com) dedicated to solving common practical cases. They provide decision support to people in need of care, employees, social workers, and consultants and can be used for systematization, categorization, and solution assignment. However, the systematization itself as well as the formulation of low-threshold accessible answers must be implemented in the future.

\section{RELATED WORK}

The techniques described in the following section are the basis for the aggregation of system components presented in the main part of this work.

\section{A. Artificial intelligence}

Artificial intelligence comprises a broad portfolio of methods such as knowledge representation, reasoning, inference, problem strategies, expert and consulting support systems with explanatory components, speech comprehension, and machine learning. While symbolic AI dominated in the 1970s until the turn of the millennium, and the breakthrough of agentbased AI models in the 1990s opened up access to "autonomisation" and thus also to intelligent human-technology interaction for all kinds of application systems, classifiers with deep learning capabilities have recently been in the foreground. Particular strengths are attributed to symbolic AI, especially with regard to the reusability of knowledge models and the traceability of derived results. Of particular importance is the AI's experience with the possibilities and, above all, the limitations of modelling and concluding evaluation of vague, uncertain, incomplete, erroneous, and missing knowledge [9]. Machine learning enables technical systems to acquire knowledge independently, to adapt existing knowledge to new experiences, and thus to adapt independently and highly flexibly to changing situational requirements, such as changes in human interaction behaviour caused by experience learning. Due to these advantages, the use of AI is also a decisive guide for the economy and administration. Central to pattern recognition methods is data pre-processing and integration by means of AI-based identification of relevant features through feature engineering. Current methods for this are, for example, deep learning based autoencoders, which train neural networks in such a way that the attributes and elements that have the greatest possible influence on the creation of the knowledge model are identified [10], [11]. A second focus of problem solving with AI is reasoning and the necessary problem solving strategies [12], [13], [14]. Further topics of current AI research are the representation of high-dimensional data [15], [16] or projection methods [17], [18] or the application possibilities of different types of neural networks [19], [20], 
[21]. The processing of natural language, e.g., in chatbots, is a necessary technique at the forefront of AI innovations.

\section{B. Text Classification}

The text classification of legally relevant passages in brochures, forms, guidebooks, and web content can be carried out using automated procedures. Up to now, Support Vector Machines have been used, whose performance was superior to other methods in this task [22]. Recent studies have shown neural networks to be promising and state of the art Numerous development steps have made it possible to make them available for a broad field of application [23], [24]. In addition, language models have recently been presented that have consistently improved the performance of neural classification tasks [25], [26]. The implementation of the intended automatic annotation of action guidelines for the classification of legally relevant text passages seems to be very promising.

\section{AI and application systems in the context of social services}

Chatbots act as virtual assistants, which are text-based dialog systems capable of mediating interaction between humans and machines in natural language and be equipped with the following features [27]:

1) filtering out superfluous information e.g., fillers, pauses, false starts;

2) determining an appropriate system response and the need for sophisticated decision making mechanism;

3) answer aggregation to present a summary answer rather than a just ranked list of results;

4) conversation management that considers and maintains the search goals, conversational history, and current state of the agent's understanding;

5) general knowledge that the agent should have about external world in order to efficiently exploit contextual information and correctly process the user's query;

6) and personality and moral responsibility to respond to emotional and sensitive queries.

A distinction can be made between rule-based chatbots and AI-based chatbots, which enable independent learning [1]. The continuous research and development of chatbots already enables their everyday use in many areas, e.g., in customer communication in e-commerce [28] or the financial sector [29]. In contrast, AI-based consulting on social services has not yet been researched [30].

\section{Regulatory hurdles of AI deployment}

Little research exists on the framework conditions for the use of an AI-based information application on legal issues. On the one hand, the particular ethical, legal, and social aspects of the new technology need to be worked out. The duties of care in programming, training, and use of machine learning systems have only been partially considered [31]. The High Level Expert Group on Artificial Intelligence (HEG-AI) of the EU Commission, which recently drafted guidelines, is investigating the framework conditions for an ethically and legally acceptable use of artificial intelligence [32]. Individual case studies on privacy and data protection aspects can only be found in blog posts by data protectionists [33].

\section{REFERENCE MODEL "PFADI.AI"}

The creation of the reference architecture in this section is the main contribution to this work. By combining proven methods and technologies of data processing, a high-level reference model, the information flows, and the technical layer of the bot are modelled and presented. Therefore the presented BERT technology serves as an enabler for the described framework.

\section{A. Methodology}

The main goal of this work relies on defining an architecture of a system that allows the integration of an AI-based chatbotsystem. For this purpose the authors propose a three-tier information system landscape "PFaDi.AI" which is based on well-proven artefacts. The presented work is based on the aggregation and combination of existing system artefacts to a new reference architecture. The creation of the model is based on the design techniques introduced by vom Brocke [34]. On a first higher layer the construction of the new architecture is presented in the next section. The following sections describe the connections of the used systems and their new arrangement to each other. Building blocks of these systems are described on a subordinate layer.

\section{B. PFaDi.AI}

The overall procedure shown in Fig. 1 of providing the information a user is searching for is described in this section. The detailed procedures of integrated components are described more precisely in the following sections. The first step in Fig. 1 starts with the initial interaction of the user with the PFaDi.AI-System. The first request is answered by the dialog management of PFaDi.AI and shows readiness for providing information. Further question and answer communication supports the definition of the topic and identifies the layer of the query space to be addressed. This is represented by steps $1-3$. Subsequently, the identified query space is used to prepare the search in the data ETL stack with the help of the BERT within the Information Retrieval. The aim is the semantic enrichment of the corpus transferred, in order to search the relevant data sinks in the following step 5 using the prepared keywords.

Following this, the identified structured or unstructured text fragments from the data sinks are prepared and broken down into processable sequences using topic modelling methods. After extracting the information from the text sequences with the help of qualifier algorithms, these sequences are brought back to Dialog Management in step 7 and made available to the user in step 8.

\section{Dialogue Management}

Dialogue Management determines the actual context of the dialogue. With respect to the features mentioned above to a 


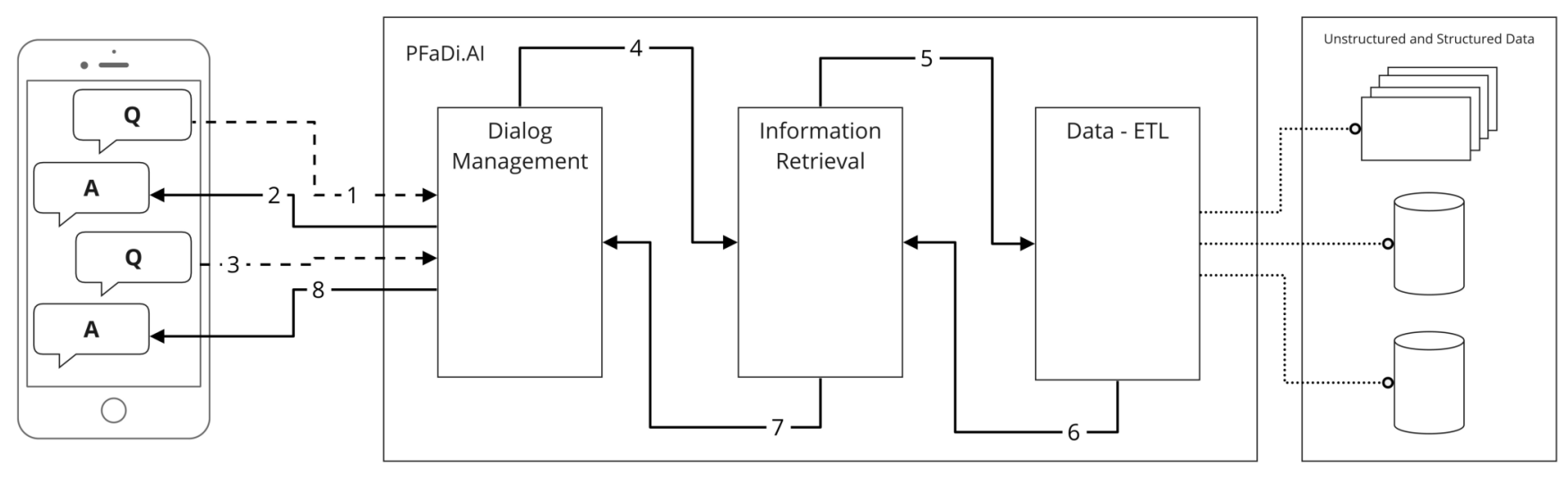

Fig. 1. High-Level Representation of the proposed architecture [own illustration]

chatbot, an architecture for an application system for social services was designed. For example, the user could say or write: "I need childcare for this week" and the bot would try to find appropriate service offerings. Then the user might add: "for the older of my two children," here the user refers to a part of the conversation that took place earlier. The bot has to correctly interpret before confirming with the user. Besides the general language, dialogue entry and exit play a special role in the conversation. When the bot starts the conversation proactively, the area of competence must be clearly defined. The definition of the dialogue exit can look manifold. It can range from pure information about the subject area of care or social services, direct booking of a service to preparing an appointment with a human consultant. An overview of the objectives of individual thematic sub-bots used in the proposed architecture is given. Based on the needs of the user, appropriate sub-bots are selected to generate high quality responses (see Table I). The individual bots are based on information transmitted by the user during the course of the conversation. The sub-bots are characterized by special domain knowledge (e.g., taxonomies and ontologies for care, paid work, and families).

TABLE I

DEFINING CHARACTERISTICS OF SUB-BOTS

\begin{tabular}{|l|l|}
\hline Name & Description \\
\hline Small Talk Bot & $\begin{array}{l}\text { By filling the bot's architecture with answers to } \\
\text { social inputs like "How Are You?, it is not only } \\
\text { prevented unnecessary fallbacks. The bot is strength- } \\
\text { ened with human qualities, which builds an invalu- } \\
\text { able connection with the user. }\end{array}$ \\
\hline Work Bot & $\begin{array}{l}\text { Answers all inquiries and provides information on } \\
\text { organizational issues within a company (e.g., swap- } \\
\text { ping shifts) as well as questions on employee rights. }\end{array}$ \\
\hline Health Care Bot & $\begin{array}{l}\text { Makes offers of care counselling and provides ap- } \\
\text { propriate information on the situation of affected } \\
\text { persons and relatives, finds services for the imple- } \\
\text { mentation of care services and gives advice when } \\
\text { applying for state support }\end{array}$ \\
\hline Family Bot & $\begin{array}{l}\text { Searches for offers of family related services. These } \\
\text { can be, for example, child care, animal care, do- } \\
\text { mestic help. An automatic booking of these services } \\
\text { should be possible. }\end{array}$ \\
\hline
\end{tabular}

The information flow of the chatbot-system is represented in Fig. 2. Users can chat with the bot not only via free text input but also speech-controlled. This is done via low-threshold use of common end devices. It's also possible to guide users through the dialogue with the help of buttons and ready-made story lines. This prevents questions being formulated in a way that is incomprehensible to the bot. No matter how well the bot is planned, it could always happen that it cannot answer a question. In this critical case, there are various escalation options: For example, a consultant can step in and take over the chat, the user can be referred to the telephone service, fill out a contact form or receive helpful documents for download. This should not result in any additional effort, otherwise the bot will not be perceived as a helper. In order to ensure targeted communication and avoid escalations the dialogue management must have mechanisms for processing feedback and policy learning. On the one hand, the agent takes the user's feedback to determine whether the bot is coping well with the conversation and whether the user is satisfied with the bot's responses. This encourages the bot to learn from mistakes and correct itself in future conversations. Through policy learning, the bot can be taught a positive language. The goal is to improve the overall satisfaction of the end user.

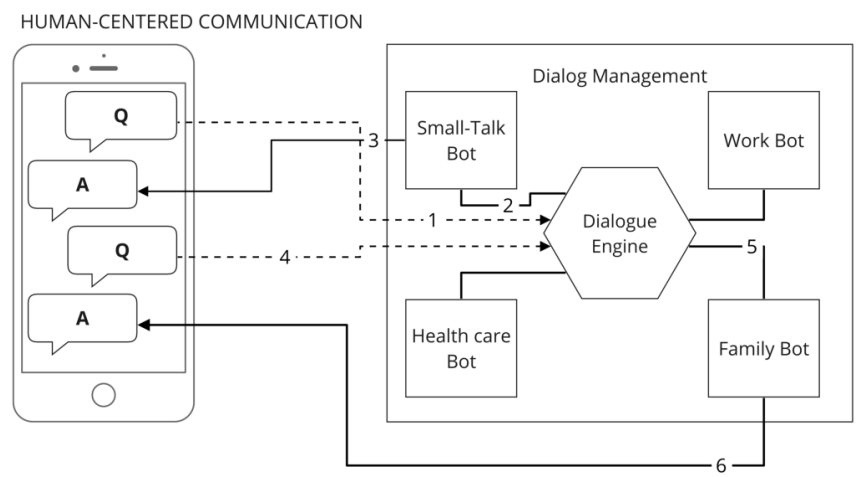

Fig. 2. Communication between human and chatbot [own illustration] 
Thus a network of successful paths has been derived and the bot is able to conduct the conversation to the satisfaction of the end user. The bot tries to learn from the interaction and to follow in detail the course of the conversation it had with similar users in the past. The technique of reinforcement learning can be used for this.

\section{Information Retrievel}

The system is placed in the area of information retrieval prepare information for further processing in subsequent systems in order to provide purposive information. Two information directions are addressed. On the one hand, the transferred question-answer constructs of the chatbots are prepared by an intent clearifier and then semantically processed by the BERT algorithm. On the other hand, sequences of answers are cut from identified relevant documents from the ELK-Stack for the transmission to the chatbot-system.

1) BERT: In the model, the BERT algorithm performs the task of providing the actual answer to the user's question. For this purpose, the user receives a content-related pre-selected sequence from the ELK, which is then searched and evaluated for the detailed answer to the user's question. As BERT is one of the core components of the system, it is discussed in more detail in chapter V. Here the technical aspects of BERT, such as the input / output representation of questions and answers or the pre-training and fine-tuning of BERT, will be discussed.

2) Intent Clearifier: The recognition of the intention of the questioner is helpful for the effective generation of an appropriate answer. For this purpose, Language Understanding Tools such as LUIS can be used. Fig. 3 shows an example of how to determine the probability of an intent to a user's question:

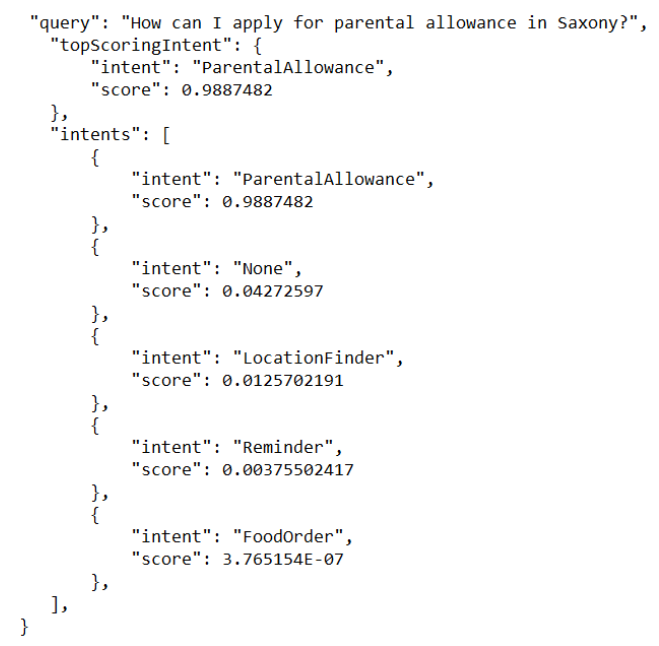

Fig. 3. Intent Clearification [own illustration]

3) Topic Modelling: In the model, communication with the user is controlled on the basis of a question and answer qualifier. This is intended to ensure that the question qualifier has all the information needed to answer the question. Here, sufficient keywords must be available to delimit the content of the topic area. On the other hand, the answer qualifier is intended to ensure that the user is only provided with information in the chatbot that exceeds at least one threshold value in the probability of correctly answering his question. Within the presented architecture, a topic modelling framework performs the task of deriving the required information from the information sinks identified by the crawler. For this purpose, the entire web page stored in the ELK stack is first segregated into paragraphs using a topic-modeling procedure. Afterwards, the paragraphs with the highest similarity to the question are made available for answering to the corresponding bot.

\section{E. Data ETL}

During the data ETL step, the contents prepared by BERT are passed to the ELK stack. Within the stack, available blocks of information are searched for on the basis of the identified subject areas and passed to the information retrieval. The information blocks are cyclically updated by a crawler ecosystem and stored in the ELK stack as shown in Fig. 4. While step 3 is triggered per each request, steps 6 and 7 are performed periodically.

1) Crawler Ecosystem: A web crawler is used to programmatically go over a selection of websites and extract its data. Such a crawler allows you to create a large text corpus from selected web pages. For the purposes of this work, the official websites on reconciling family and paid work at EU level and at federal and state level will be searched in German language (e.g., https://www.bmfsfj.de/). For a corresponding specialisation of the information, additional company-specific information can be added to the text corpus.

As an application framework scrappy is used to crawl these websites for structured (e.g., HTML) and unstructured data (e.g., PDF). You can get the HTML source code through the scrappy downloader.

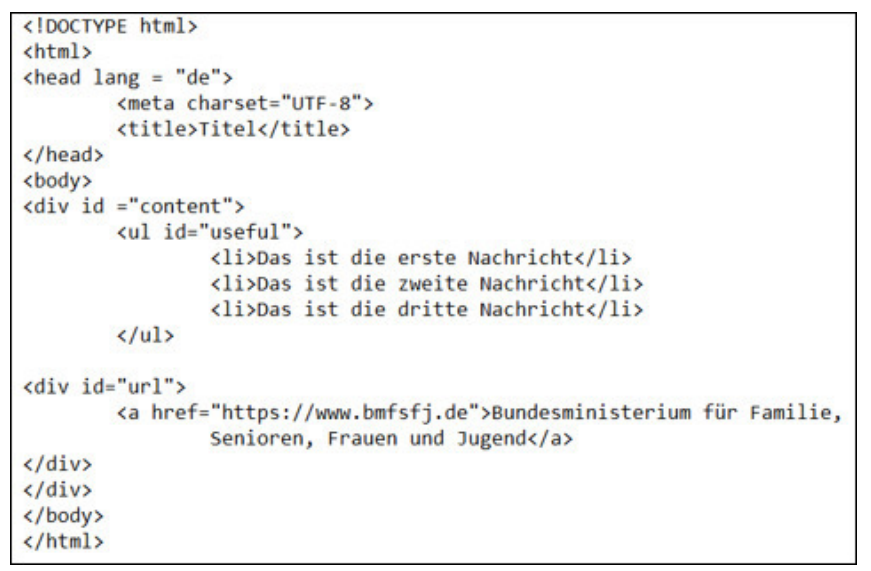

Fig. 5. HTML source code of a simple web page [own illustration]

The scrappy downloader can access the web data according to the specified URL. With selectors certain parts of the HTML document can be specified by Xpath expressions. For the 


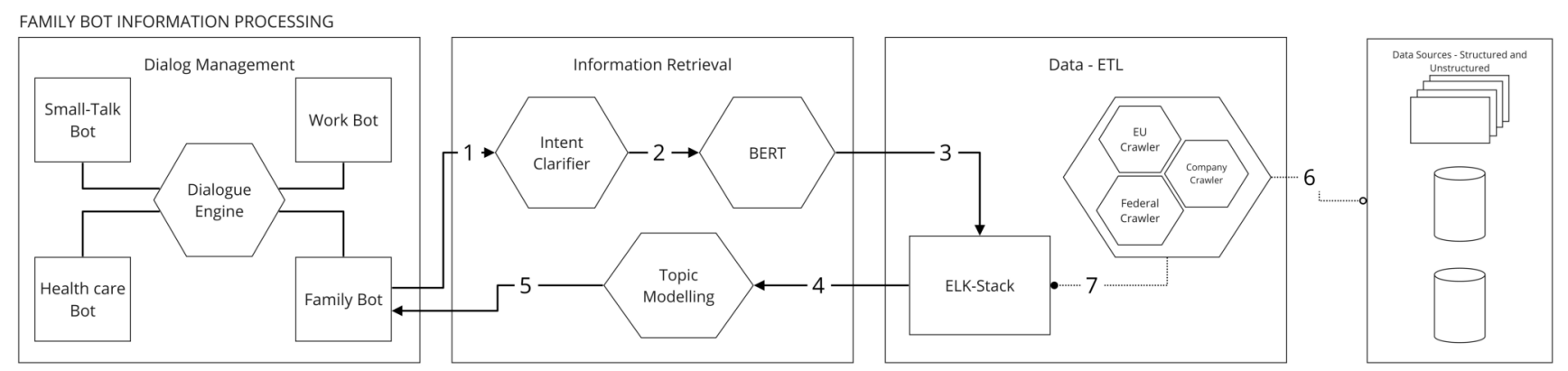

Fig. 4. Information flow - technical layer with communication between system aggregates [own illustration]

HTML source code in Fig. 5 you can construct an Xpath for selecting the text under the label and extracting the text inside the attribute from the HTML source code.

2) ELK-Stack: This ELK stack is used to collect and store the structured and unstructured data generated by the web crawler. Furthermore, the document-oriented database Elasticsearch is used to retrieve the collected information for the purpose of finding an appropriate response. The Elastic or "ELK" stack contains three open source tools: Elasticsearch, Logstash, and Kibana. It is a document-oriented database designed to store, retrieve, and manage document-oriented or semi-structured data. Logstash is a server-side data processing pipeline that simultaneously ingests data from multiple sources, converts it and then sends it to a storage location, such as Elasticsearch. Kibana allows users to visualize data through charts and tables in Elasticsearch.

\section{BERT: BIDIRECTIONAL ENCODER REPRESENTATIONS FROM TRANSFORMERS}

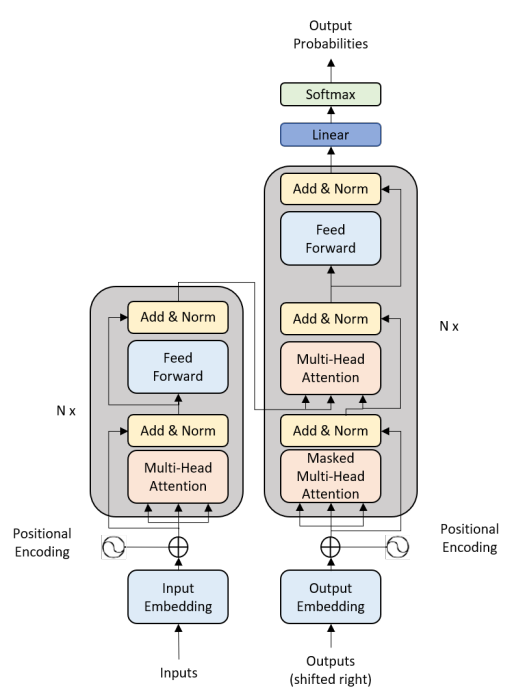

Fig. 6. Transformer Model Architecture [35]

Bidirectional Encoder Representations from Transformers (BERT) [36] represents the state of the art of language models that pretrain deep bidirectional representations for a variety of tasks. Possible fields of application include text classification, named entity recognition or question answering tasks (i. e. SQUAD 2.0) [37]. The Bert implementation is a two-stage process consisting of a pre-training step and a fine-tuning step. During the pre-training step the model is trained on unlabelled data. Based on this the fine-tuning step follows. Here the model is initialized with the pre-trained parameters, which are then fine-tuned with labelled data from downstream tasks.

\section{A. BERT Baseline Model Architecture}

BERT is based on the transformer architecture described by Vaswani et al. [35]. Fig. 6 shows a transformer model architecture. For a more intensive study of Transformer Architecture, please refer to [35].

\section{B. Input / Output Representations}

The input representation of BERT can represent both a single sentence and a pair of sentences (e.g. <Question, Answer $>$ ) in one token sequence. An example sentence could be as follows:

\section{Where can I find the nearest childcare?}

The tokinization of this sentence is then as follows:

['[CLS]', 'Where', 'can', 'I', 'find', 'the', 'nearest', 'childcare', '?', '[SEP]']

The first token of each sequence is always a special class token 'CLS'. The hidden state associated with this token is used for classification tasks as an aggregated sequence representation. For a pair of sentences within a sequence another special token 'SEP' is used. This token is used to separate the individual sentences. For a question-answer task the following structure can be used:

['CLS', ' $q w 1$ ', ... ' $q w N$ ', 'SEP', 'cwl', . . . ., 'c $c w M$ ', 'SEP']

It can be used this input representation for each sequence. Here 'CLS' stands for a classification token and 'SEP' for a separation token. Furthermore 'qw' represent a question word 
with $\mathrm{N}$, the number of question words and ' $\mathrm{cw}$ ' represents a context word with $\mathrm{M}$, the number of context words. The representations are taken by the first encoder transformer and retrieve embeddings for each of the words. The input embeddings are the sum of the token embeddings, the segmentation embeddings, and the position embeddings.

\section{PRE-TRAINING BERT}

Instead of using traditional left-to-right or right-to-left language models [38][39], it can be used as an unsupervised tasks to pre-train BERT. For the purpose of Question Answering and Natural Language Inference the Next Sentence Prediction task is described in this section.

\section{A. Next Sentence Prediction}

For downstream tasks like Question Answering (QA) it is important to understand the relationships between two sentences, which is not directly captured by language models. For that reason BERT is pre-trained for a binarized next sentence prediction task. This task can be generated from any monolingual corpus. $50 \%$ of the time $\mathrm{B}$ is the actual next sentence that follows A (labeled as isNext) when choosing the sentences A and B for each pre-training example. For the other $50 \%$ it is a random sentence from the corpus (labeled as NotNext). The following example illustrates the next sentence prediction task:

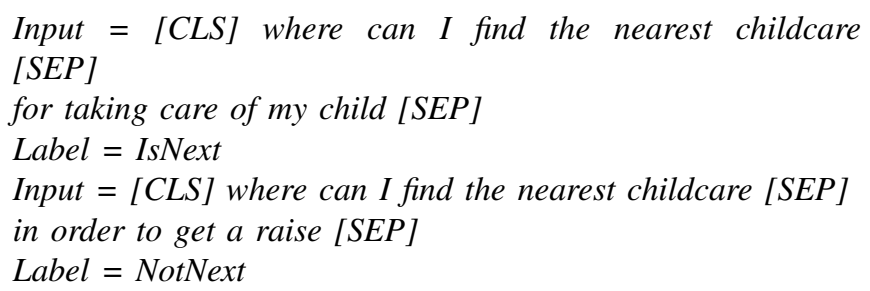

The next sentence prediction task is related to representation learning objectives used in [40] and [41].

\section{B. Pre-training and fine-tuning BERT}

For pre-training purposes the corpus data in German language is used, which was created from the text data obtained by the web crawler.

BERT uses the self-attention mechanism to unify the encoding of the text pairs as well as the application of the bidirectional cross attention between concatenated sentences. Because of that fine-tuning is a straightforward task. For fine-tuning the task-specific inputs and outputs are simply plugged into BERT and an end-to-end fine-tuning of all parameters is performed. At the input, sentence $\mathrm{A}$ and sentence $\mathrm{B}$ from pre-training are analogous to question-passage pairs in question answering. At the output, the token representations are fed into an output layer for token level tasks, e.g., question answering, and the [CLS] representation is fed into an output layer for classification. The hyperparameter-tuning includes setting an appropriate batch-size, learning rate or number of epochs and is very task specific.

\section{CONCLUSiOn AND Future WORK}

In this work a conception of an AI based assistant to support the compatibility of family and paid work was presented. For this purpose, the essential components of the bot from data generation, data storage, and data flows to information retrieval and data management were described. The main component of the system is represented by BERT, which contributes to the classification of user questions in terms of content and thus to a more optimised finding of answers by the sub-bots. The presented architecture components are state of the art components from the areas of data pipelines, data processing, and artificial intelligence which have been aggregated and combined into a new reference architecture and thus answer the research question posed at the beginning about the appearance of a suitable approach for a chatbot architecture for a better compatibility of family and paid work. This presented preliminary approach for a chatbot architecture for better reconciliation of family and paid work, especially in the care and health sector, is a promising starting point for further developments. The bot is supposed to be used by the user via specific end devices (e.g., smart phone, tablet, pc) based on speech or text. Whereby the use should be as low-threshold as possible. In a next step, the reference model and its components will be implemented in a first prototype with the help of the latest technologies from science and engineering. This is to be used initially as a prototype and then productively in the above-mentioned areas in order to mitigate the effects of the corona pandemic on the compatibility of family and paid work and to better master future challenges. By integrating PFaDi.AI into the PFaDi platform, the aim is to enable employees to structure their daily lives efficiently through easily accessible information and services and to minimise the impact of the corona pandemic on the reconciliation of paid work and family life. For this reason it is necessary to implement the described steps as quickly as possible and to present them to the scientific community in order to discuss the presented approach and raise additional potential.

\section{REFERENCES}

[1] H. N. Io and C. B. Lee, "Chatbots and conversational agents: A bibliometric analysis," in 2017 IEEE International Conference on Industrial Engineering and Engineering Management (IEEM), 2017, pp. 215-219.

[2] P. Böttinger and J. zu Putlitz, Die Zukunft der Medizin: Disruptive Innovationen revolutionieren Medizin und Gesundheit. Mit einem Geleitwort von Hasso Plattner. Berlin: MWV Medizinisch Wiss. Ver, 2019. [Online]. Available: https://books.google.de/books?id=42rKDwAAQBAJ

[3] F. Resch and P. Parzer, Entwicklungspsychopathologie und Psychotherapie - Kybernetische Modelle zur funktionellen Diagnostik bei Jugendlichen. Berlin Heidelberg New York: Springer-Verlag, 2015.

[4] S. Kaiser and M. J. Ringlstetter, Work-Life Balance - Erfolgversprechende Konzepte und Instrumente für Extremjobber. Berlin Heidelberg New York: Springer-Verlag, 2010.

[5] S. Peters and J. v. Garrel, Arbeits - Zeitsouveränität für Führungskräfte von Morgen - Vereinbarkeit von Beruf und Privatheit. München: Rainer Hampp Verlag, 2013.

[6] M. Boniol, M. McIsaac, L. Xu, T. Wuliji, D. K., and J. Campbell, "Gender equity in the health workforce: Analysis of 104 countries." Health Workforce Working paper 1., 2019. 
[Online]. Available: https://apps.who.int/iris/bitstream/handle/10665/ 311314/WHO-HIS-HWF-Gender-WP1-2019.1-eng.pdf?ua=1

[7] M. Lukasczik et al., Compatibility of Work and Family Life of Employees in the Healthcare Sector: An Issue in Health Services Research. Gesundheitswesen (Bundesverband der Ärzte des Öffentlichen Gesundheitsdienstes (Germany), 2013.

[8] K. P. Jerg-Bretzke, L., H. C. Traue, and K. Limbrecht-Ecklundt, "What you will": Results of an Empirical Analysis of the Need to Improve Work-life Balance for Physicians. Berlin: MWV Medizinisch Wiss. Ver, 2018.

[9] S. Russell and P. Norvig, Artificial Intelligence: A Modern Approach, Global Edition. CL, may 2019. [Online]. Available: https://www.xarg. org/ref/a/1292153962/

[10] T. Nolle, S. Luettgen, A. Seeliger, and M. Mühlhäuser, "Analyzing business process anomalies using autoencoders," Mach. Learn., vol. 107, no. 11, p. 1875-1893, Nov. 2018. [Online]. Available: https://doi.org/10.1007/s 10994-018-5702-8

[11] C. Zhou and R. C. Paffenroth, "Anomaly detection with robust deep autoencoders," in Proceedings of the 23rd ACM SIGKDD International Conference on Knowledge Discovery and Data Mining, ser. KDD '17. New York, NY, USA: Association for Computing Machinery, 2017, p. 665-674. [Online]. Available: https://doi.org/10.1145/3097983.3098052

[12] L. Barreto and E. Mota, "Self-organized inductive reasoning with nemus," CoRR, vol. abs/1906.06761, 2019. [Online]. Available: http://arxiv.org/abs/1906.06761

[13] Y. Jiang and M. Bansal, "Avoiding reasoning shortcuts: Adversarial evaluation, training, and model development for multi-hop QA," CoRR, vol. abs/1906.07132, 2019. [Online]. Available: http://arxiv.org/abs/ 1906.07132

[14] M. Qu and J. Tang, "Probabilistic logic neural networks for reasoning," CoRR, vol. abs/1906.08495, 2019. [Online]. Available: http://arxiv.org/abs/1906.08495

[15] S. Liu, D. Maljovec, B. Wang, P. Bremer, and V. Pascucci, "Visualizing high-dimensional data: Advances in the past decade," IEEE Transactions on Visualization and Computer Graphics, vol. 23, no. 3, pp. 1249-1268, 2017.

[16] C. Seifert et al., Visualizations of Deep Neural Networks in Computer Vision: A Survey, ser. Studies in Big Data. Springer, 2017, pp. 123-144.

[17] P. Oesterling, C. Heine, H. Janicke, G. Scheuermann, and G. Heyer, "Visualization of high-dimensional point clouds using their density distribution's topology," IEEE Transactions on Visualization and Computer Graphics, vol. 17, no. 11, pp. 1547-1559, 2011.

[18] C. Heine et al., "A survey of topology-based methods in visualization," Comput. Graph. Forum, vol. 35, no. 3, p. 643-667, Jun. 2016.

[19] J. Wang, L. Gou, H. Shen, and H. Yang, "Dqnviz: A visual analytics approach to understand deep q-networks," IEEE Transactions on Visualization and Computer Graphics, vol. 25, no. 1, pp. 288-298, 2019.

[20] B. C. Kwon et al., "Retainvis: Visual analytics with interpretable and interactive recurrent neural networks on electronic medical records," CoRR, vol. abs/1805.10724, 2018. [Online]. Available: http://arxiv.org/abs/1805.10724

[21] V. Dibia and C.. Demiralp, "Data2vis: Automatic generation of data visualizations using sequence to sequence recurrent neural networks," CoRR, vol. abs/1804.03126, 2018. [Online]. Available: http://arxiv.org/abs/1804.03126

[22] A. Niekler et al., "ILCM - a virtual research infrastructure for largescale qualitative data," in Proceedings of the Eleventh International Conference on Language Resources and Evaluation (LREC 2018). Miyazaki, Japan: European Language Resources Association (ELRA) May 2018. [Online]. Available: https://www.aclweb.org/anthology/ L18-1209

[23] A. Jacovi, O. Sar Shalom, and Y. Goldberg, "Understanding convolutional neural networks for text classification," in Proceedings of the 2018 EMNLP Workshop BlackboxNLP: Analyzing and Interpreting
Neural Networks for NLP. Brussels, Belgium: Association for Computational Linguistics, Nov. 2018, pp. 56-65. [Online]. Available: https://www.aclweb.org/anthology/W18-5408

[24] S. Lai, L. Xu, K. Liu, and J. Zhao, "Recurrent convolutional neural networks for text classification," in AAAI, 2015.

[25] J. Devlin, M. Chang, K. Lee, and K. Toutanova, "BERT: pre-training of deep bidirectional transformers for language understanding," CoRR, vol. abs/1810.04805, 2018. [Online]. Available: http://arxiv.org/abs/ 1810.04805

[26] J. Li, A. Sun, J. Han, and C. Li, "A survey on deep learning for named entity recognition," CoRR, vol. abs/1812.09449, 2018. [Online]. Available: http://arxiv.org/abs/1812.09449

[27] L. Azzopardi, M. Dubiel, M. Halvey, and J. Dalton, "Conceptualizing agent-human interactions during the conversational search process," 2018.

[28] L. Cui, S. Huang, F. Wei, C. Tan, C. Duan, and M. Zhou, "SuperAgent: A customer service chatbot for e-commerce websites," in Proceedings of ACL 2017, System Demonstrations. Vancouver, Canada: Association for Computational Linguistics, Jul. 2017, pp. 97-102. [Online]. Available: https://www.aclweb.org/anthology/P17-4017

[29] T. Okuda and S. Shoda, "Ai-based chatbot service for financial industry," Fujitsu Scientific and Technical Journal, vol. 54, no. 2, pp. 4-8, 2018.

[30] E. H. Almansor and F. K. Hussain, "Survey on intelligent chatbots: State-of-the-art and future research directions," in CISIS, 2019.

[31] N. Bilski and T. Schmid, "Verantwortungsfindung beim einsatz maschinell lernender systeme, njoz 2019, 657-661," vol. 19, p. 657, 052019.

[32] B. J. Wagner, "Rechtliche Analyse der Kommunikationsströme zwischen Assistenzsystemen," in Konnektivität von Assistenzsystemen: Eine vertrags- und datenschutzrechtliche Analyse kollaborativer Softwareagenten im Internet of Things. Wiesbaden: Springer Fachmedien Wiesbaden, 2020, pp. 65-436. [Online]. Available: https://doi.org/10.1007/978-3-658-29628-5_3

[33] H. Harkous, K. Fawaz, K. G. Shin, and K. Aberer, "Pribots: Conversational privacy with chatbots," in Twelfth Symposium on Usable Privacy and Security ( $\{$ SOUPS $\}$ 2016), 2016.

[34] J. v. Brocke, Konstruktionstechniken zur Referenzmodellierung - Enzyklopaedie der Wirtschaftsinformatik, 012018.

[35] A. Vaswani et al., "Attention is all you need," CoRR, vol. abs/1706.03762, 2017. [Online]. Available: http://arxiv.org/abs/1706 03762

[36] J. Devlin, M. W. Chang, K. Lee, and K. Toutanova, "BERT: pre-training of deep bidirectional transformers for language understanding," CoRR, vol. abs/1810.04805, 2018. [Online]. Available: http://arxiv.org/abs/ 1810.04805

[37] D. Takeuchi and K. Tran, "Improving squad 2.0 performance using bert + x," 32nd Conference on Neural Information Processing Systems (NIPS), vol. abs/1810.04805, 2018. [Online]. Available: http://arxiv.org/abs/1810.04805

[38] M. E. Peters et al., "Deep contextualized word representations," CoRR, vol. abs/1802.05365, 2018. [Online]. Available: http://arxiv.org/abs/ 1802.05365

[39] A. Radford, K. Narasimhan, T. Salimans, and I. Sutskever, "Improving language understanding by generative pre-training," https://cdn.openai.com/research-covers/language-unsupervised/ language_understanding_paper.pdf, 2018.

[40] Y. Jernite, S. R. Bowman, and D. A. Sontag, "Discourse-based objectives for fast unsupervised sentence representation learning," CoRR, vol. abs/1705.00557, 2017. [Online]. Available: http://arxiv.org/ abs/1705.00557

[41] L. Logeswaran and H. Lee, "An efficient framework for learning sentence representations," CoRR, vol. abs/1803.02893, 2018. [Online]. Available: http://arxiv.org/abs/1803.02893 